\title{
Preliminary Validation and Feasibility of the Autism Detection in Early Childhood-Virtual (ADEC-V) for Autism Telehealth Evaluations in a Hospital Setting
}

\author{
Elizabeth M. Kryszak ${ }^{1,2,6}$ - Charles M. Albright ${ }^{1,2} \cdot K^{2}$ Kevin G. Stephenson ${ }^{1,2} \cdot$ Rose E. Nevill $^{3} \cdot$ Darren Hedley $^{4}$. \\ Claire O. Burns ${ }^{1} \cdot$ Robyn L. Young $^{5} \cdot$ Eric M. Butter $^{1,2} \cdot$ Kerrigan Vargo $^{1} \cdot$ James A. Mulick $^{2}$
}

Accepted: 5 January 2022 / Published online: 9 February 2022

(c) The Author(s), under exclusive licence to Springer Science+Business Media, LLC, part of Springer Nature 2022

\begin{abstract}
This study provided preliminary validation of the Autism Detection in Early Childhood-Virtual (ADEC-V) for telehealth assessment of possible autism. Participants were 121 children (24.79\% female) aged 18-47 months who completed telehealth evaluations at a large pediatric hospital in the Midwestern United States between October 2020 and February 2021. The ADEC-V showed good sensitivity (0.82) and specificity (0.78) and was significantly correlated with other ASD symptom measures (i.e., CARS-2, ADI-R). Internal consistency was acceptable $(\alpha=0.77)$. These results need replication in a larger and broader sample including more children without ASD. This preliminary validation study identifies the ADEC-V as a promising measure for telehealth ASD assessments in young children.
\end{abstract}

Keywords Autism spectrum disorder $\cdot$ Telemedicine $\cdot$ Assessment $\cdot$ Screening $\cdot$ Young children $\cdot$ Psychometrics

\section{Introduction}

Preliminary validation and feasibility of the Autism Detection in Early Childhood-Virtual (ADEC-V) for Autism Telehealth Evaluations in a Hospital Setting.

The COVID-19 pandemic has highlighted the need to develop effective, evidence-based models for delivering Autism Spectrum Disorder (ASD) diagnostic services remotely, such as through telehealth. Best practice guidelines

Elizabeth M. Kryszak

Elizabeth.kryszak@nationwidechildrens.org

Nationwide Children's Hospital, Columbus, OH, USA

2 Department of Pediatrics and Psychology, The Ohio State University, Columbus, $\mathrm{OH}$, USA

3 School of Education and Human Development, University of Virginia, Charlottesville, VA, USA

4 School of Psychology \& Public Health, Olga Tennison Autism Research Centre, La Trobe University, Melbourne, VIC, Australia

5 Flinders University, Bedford Park, SA, Australia

6 Child Development Center, Nationwide Children's Hospital, 187 W. Schrock Rd., Westerville, OH 43081, USA for autism assessment and diagnosis include the use of goldstandard measures such as the Autism Diagnostic Observation Schedule, Second Edition (ADOS-2; Lord et al., 2012). Assessments completed via telehealth present increased challenges to use of traditional or gold standard assessment procedures, including reducing the clinician's control of the assessment environment, an increased reliance on parents to apply prompts, limited visibility of the child or environment during the meeting, and fewer opportunities for the clinician to directly engage with or respond to the patient. Berger et al. (2021) also emphasized the impact of difficulties with internet connectivity and potential lack of access to necessary materials, thereby highlighting the risk of additional barriers to families who are economically disadvantaged. Many existing semi-structured behavioral observation measures require in-person administration and are consequently not currently suited for telehealth. Therefore, there is an urgent need to develop new assessment instruments, or modify existing instruments, that can be applied in a telehealth setting. These types of measures may serve to address barriers beyond the current pandemic restrictions that tend to affect families' ability to access in-clinic services. Some of these barriers include living in remote 
or rural areas, access to specialist clinicians (Zwaigenbaum $\&$ Warren, 2021), expense of the evaluation, length of the assessment, transportation issues, or childcare difficulties (Gordon-Lipkin et al., 2016). The Autism Detection in Early Childhood (ADEC; Young, 2007) is a brief behavioral observation measure with strong research support for the detection and diagnosis of young children suspected of ASD (Hedley et al., 2015; Nah et al., 2014, 2019; Young \& Nah, 2016). The present study describes the preliminary validation and feasibility of using the ADEC in a telehealth setting.

If telehealth is to be used as a suitable alternate or adjunct to face-to-face ASD assessments, then to comply with bestpractice guidelines, semi-structured behavioral observation tools must be valid and reliable for use over this medium. In a recently published review, Berger et al. (2021) identified five tools for conducting virtual ASD assessments in toddlers (i.e., 12-36 months of age) via observation of semistructured activities with the parent under the direction of a clinician. These measures included the Autism Spectrum Disorder-Diagnostic Interview and Activities, Lifespan, Version 2 (ASD-DIAL; Miller, 2020), the Autism Detection in Early Childhood-Virtual (Kryszak \& Albright, 2020; Young, 2007), Brief Observation of Symptoms of Autism (BOSA; Lord et al., 2020), Observation of Play Screener: Home Edition (OOPS:HE; Nickel, 2020), and the TELEASD-PEDS (Corona et al., 2020a, b). A recent multi-site study encouraged the use of a hybrid approach to assessment, describing the telehealth behavioral assessment portion of the evaluation as including one of the following measures: the TELE-ASD-PEDS, Childhood Autism Rating Scale, Second Edition (CARS-2; Schopler et al., 2010), or the Systematic Observation of Red Flags of Autism Spectrum Disorder (SORF; Wetherby et al., 2004), as well as questions and activities from the BOSA and/or ADOS-2, based on the clinical presentation of the patient (Jang et al., 2021).

One of these virtual assessments for ASD in young children that is currently widely used is the TELE-ASD-PEDS, which is based on the Screening Tool for Autism in Toddlers and Young Children (STAT; Stone et al., 2000). The TELE-ASD-PEDS has feasibility for clinical use and utility for providing valuable clinical information in the context of the broader assessment (Corona et al., 2020b; Wagner et al., 2021). Despite evidence for its clinical utility, evaluation of the accuracy, reliability, and concurrent validity of the TELE-ASD-PEDS has yet to be fully established (Wagner et al., 2020).

Whereas several of the measures previously discussed were developed specifically for use virtually, they also have not yet been established as reliable and valid (Corona et al., 2020b; Lord et al., 2020; Nickel, 2020). Another approach to addressing the need for virtual assessment tools is to modify short, easy-to-use tools with established psychometric properties for telehealth use. The ADEC (Young, 2007), originally designed as a brief in-person observation tool, has well-established psychometric properties (Young, 2007), strong research support for use to accelerate and inform clinical screening for ASD (Hedley et al., 2015; Young \& Nah, 2016), and its scores show very good reliability with the ADOS-2 (Hedley et al., 2015; Bellando et al., 2018). It is a published Level 2 screening tool that is time-efficient, suitable for children under 3 years, easy to administer, and requires minimal training and experience to administer reliably. As such, it lends itself easily to the transition to an online platform. The ADEC has further demonstrated robust clinical properties when abbreviated to a five-item format, which has potential for application in settings such as during child wellness checks (Nah et al., 2019; Nevill et al., 2019). The ADEC has been translated and validated for use in Spanish (Hedley et al., 2010), Japanese (Kawashima et al., 2021), Indonesian (Sidjaja, 2015), and has been piloted for use in Singapore (Nah et al., 2014). It has been found to perform similarly to the ADI-R and CARS (Nah et al., 2014) as well as the ADOS-2, particularly the toddler module (Hedley et al., 2015), in differentiating ASD from other conditions in toddlers.

Therefore, given the significant body of research support, the ADEC has potential utility for use as a semi-structured behavioral observation for telehealth. Aspects of the instrument that would make it amenable to this application include that it is brief (20-25 min to administer by telehealth), easy to learn and achieve reliability, and implements a simple coding system with clear behavioral anchors. Additionally, materials required for the ADEC do not need to be purchased from the publisher's website and include toys and items that are typically found in a family home (e.g., toy cars, stacking cups). The simplicity of the items suggests straightforward administration, whereby a clinician would be able to coach a caregiver to administer the items while the clinician observes and scores the items. Therefore, telehealth administration would require few modifications to the standardized administration protocol to be used in a telehealth setting. Compared to other measures, such as the TELEASD-PEDS, the ADEC protocol provides more specific scoring guidelines for several of the items (which is similar to the way many items on the ADOS-2 are scored; Lord et al., 2012), and also includes additional deliberate prompts for specific behaviors; for example, imitation, response to specific sounds, and rigidity regarding the placement of objects (Young, 2007).

\section{Aims of Current Study}

The primary aim of the current study was to investigate the clinical utility, validity, and reliability of a modified version of the ADEC, the ADEC-Virtual (ADEC-V), 
for telehealth ASD evaluations of young children. The ADEC-V was examined in a clinical environment at one of the largest behavioral health departments in the United States (US). The current study had four primary hypotheses: (1a) Predictive Validity: The virtual (telehealth) adaptation of the Autism Detection in Early Childhood (ADEC-V) will have acceptable diagnostic accuracy (i.e., sensitivity and specificity $>0.80$ ) in distinguishing children three years old and younger when compared to best estimate clinical (BEC) diagnosis of autism spectrum disorder (ASD) using all available clinical information, excepting ADEC-V scores; (1b) Diagnostic Validity: Using logistic regression analysis, ADEC-V scores will significantly predict BEC diagnosis after controlling for age and developmental level (e.g., Developmental Profile4th Edition [DP-4] scores); (2) Concurrent Validity: Final score on the ADEC-V will be significantly correlated with ADI-R and CARS-2; (3) Internal consistency: Reliability of ADEC-V items will be acceptable (i.e., McDonald's omega $\geq 0.80$ ).

\section{Method}

\section{Participants}

Participants were 121 children ages $18-47$ months ( $M=34.40, S D=7.02$ months, $24.79 \%$ female) who completed a telehealth evaluation with their caregivers through a developmental assessment center at a large pediatric hospital in the Midwestern United States between October 2020 and February 2021. Children were referred for a(n) uni- or interdisciplinary autism diagnostic assessment after completing a preliminary unstructured screening clinical interview and were deemed at risk for ASD. A large portion of the sample lived in rural areas $(\mathrm{n}=37,30.58 \%)$ and had state (Medicaid) insurance $(\mathrm{n}=79,65.29 \%)$. The sample was racially diverse (57.02\% White, $19.83 \%$ Black, $20.66 \%$ other). Most of the participants were given a final diagnosis of ASD $(n=103$, $85.12 \%)$. For the 18 participants who did not receive an ASD diagnosis, the primary diagnosis was global developmental delay $(n=11)$ or a language disorder $(n=7)$. The two diagnostic groups (children with versus without ASD) did not differ in age [ $t(24.64)=0.52, p=0.61]$, biological sex [McNemar's $\chi^{2}(1)=3.025, p=0.08$ ], race $\left[\chi^{2}(2)=0.90, p=0.64\right]$, or rural status $\left[\chi^{2}(1)=1.22\right.$, $p=0.27]$. The ASD group was more likely than the nonASD group to have non-Medicaid insurance $[38.83 \%$ vs. $\left.11.11 \% ; \chi^{2}(1)=4.05, p=0.04\right]$ (see Table 1$)$.

\section{Measures}

\section{Autism Detection in Early Childhood-Virtual (ADEC-V)}

The ADEC (Young, 2007) is a validated, 16-item level 2 screener of behaviors characteristic of autism in children ages 12-36 months old, which are often absent or atypical in children with ASD. It is behavior-based and interactive. Items are coded from 0 to 2 , with 0 implying an age-appropriate response, 1 indicating a somewhat inappropriate response, and 2 indicating a clearly inappropriate response. Risk for ASD is determined based on ADEC Total scores, and is determined as low (total scores from 0 to 10$)$, moderate (11-13), high (14-19) or very high (>19). It can be administered in 10-15 min.

The ADEC-V is a modification of the original tool developed to facilitate virtual administration by caregivers under clinician guidance. Modifications included rewording of instructions to allow caregivers to administer all items while coached by a clinician, use of non-standardized manipulatives, and asking caregivers to report on behaviors they observed to either confirm the clinician's judgement or inform the clinician if there was a behavior the clinician was unable to observe over webcam (e.g., eye contact). In the present study, it was decided to include children up to 47 months of age. While there is some support for using the instrument in older children (Hedley et al., 2010), for the present study the decision to trial the instrument in children up to 4 years of age was primarily due to the lack of alternative instruments that could be administered remotely for this age range. Thus, if the instrument was found to be valid for use in older children it would be clinically useful for the hospital and for other clinicians evaluating ASD by telehealth using this measure. The modifications were developed with the collaboration of the author of the instrument (R.L.Y.).

\section{Autism Diagnostic Interview-Revised (ADI-R; Le Couteur et al., 2003)}

The ADI-R is a comprehensive, semi-structured clinical caregiver interview for children and adults with a mental age above 2 years that is considered a gold standard autism diagnostic tool. Administration and scoring take between 90 and $150 \mathrm{~min}$. The three areas assessed in the ADI-R include: (1) language/communication; (2) reciprocal social interactions and (3) restricted, repetitive, and stereotyped behavior and interests. The ADI-R demonstrates strong inter-rater reliability in clinical settings (Zander et al., 2017) and good construct validity in children with ASD (Lecavalier et al., 2006). Only the items included in the modified toddler algorithm (Kim \& Lord, 2012a) were administered. 
Table 1 Participant characteristics

\begin{tabular}{|c|c|c|c|c|c|}
\hline & Total $(N=121)$ & $\operatorname{ASD}(N=103)$ & Non-ASD $(N=18)$ & $\begin{array}{l}\text { Group difference } \\
\text { Statistic ( } p \text { value) }\end{array}$ & Number missing (\%) \\
\hline Age in months (mean/SD) & $34.40(7.02)$ & $34.3(7.04)$ & $35.01(7.06)$ & $t(24.64)=0.52(0.61)$ & $0(0)$ \\
\hline Biological sex & & & & $\chi^{2}(1)=3.025(0.08)^{\mathrm{a}}$ & $0(0)$ \\
\hline Female & $30(24.79 \%)$ & $26(25.24 \%)$ & $4(22.22 \%)$ & & \\
\hline Male & $91(75.21 \%)$ & $77(74.76 \%)$ & $14(77.78 \%)$ & & \\
\hline Race & & & & $\chi^{2}(2)=0.90(0.64)$ & $3(2.48)$ \\
\hline Black & $24(20.34 \%)$ & $22(21.78 \%)$ & $2(11.76 \%)$ & & \\
\hline White & $69(58.47 \%)$ & $58(57.43 \%)$ & $11(64.71 \%)$ & & \\
\hline Other & $25(21.19 \%)$ & $21(20.79 \%)$ & $4(23.53 \%)$ & & \\
\hline Rural & & & & $\chi^{2}(1)=1.22(0.27)$ & $0(0)$ \\
\hline Yes & $37(30.58 \%)$ & $29(28.16 \%)$ & $8(44.44 \%)$ & & \\
\hline No & $84(69.42 \%)$ & $74(71.84 \%)$ & $10(55.56 \%)$ & & \\
\hline Insurance & & & & $\chi^{2}(1)=4.05(0.04)$ & $0(0)$ \\
\hline Medicaid & $79(65.29 \%)$ & $63(61.17 \%)$ & $16(88.89 \%)$ & & \\
\hline Non-medicaid & $42(34.71 \%)$ & $40(38.83 \%)$ & $2(11.11 \%)$ & & \\
\hline ADEC-V total (mean/SD) & $17.66(5.98)$ & $18.86(5.45)$ & $10.78(3.90)$ & $t(29.95)=7.59(<0.001)$ & $0(0)$ \\
\hline ADI-R algorithm & & & & $\chi^{2}(2)=12.61(0.002)$ & $8(6.61)$ \\
\hline Young/nonverbal & $57(50.44 \%)$ & $54(56.25 \%)$ & $3(17.65 \%)$ & & \\
\hline Single words & $38(33.63 \%)$ & $31(32.29 \%)$ & $7(41.18 \%)$ & & \\
\hline Phrase speech & $18(15.93 \%)$ & $11(11.46 \%)$ & $7(41.18 \%)$ & & \\
\hline ADI-R category & & & & $\chi^{2}(2)=34.88(<0.001)$ & $8(6.61)$ \\
\hline Little-to-no & $16(14.16 \%)$ & $6(6.25 \%)$ & $10(58.82 \%)$ & & \\
\hline Mild-to-moderate & $25(22.12 \%)$ & $21(21.88 \%)$ & $4(23.53 \%)$ & & \\
\hline Moderate-to-severe & $72(63.72 \%)$ & $69(71.88 \%)$ & $3(17.65 \%)$ & & \\
\hline CARS- 2 category & & & & $\chi^{2}(2)=82.90(<0.001)$ & $4(3.31)$ \\
\hline Minimal-to-no symptoms & $19(16.24 \%)$ & $3(3.03 \%)$ & $16(88.89 \%)$ & & \\
\hline Mild-to-moderate symptoms & $46(39.32 \%)$ & $44(44.44 \%)$ & $2(11.11 \%)$ & & \\
\hline Severe symptoms & $52(44.44 \%)$ & $52(52.53 \%)$ & $0(0 \%)$ & & \\
\hline CARS-2 total (mean/SD) & $35.24(6.33)$ & $35.41(5.68)$ & $35.20(6.51)$ & $t(24.49)=12.14(<0.001)$ & $4(3.31)$ \\
\hline Cognitive level $^{\mathrm{b}}$ & & & & $\chi^{2}(1)=5.76(0.02)$ & $16(13.22)$ \\
\hline$\geq 70$ & $35(33.33 \%)$ & $25(28.09 \%)$ & $10(62.50 \%)$ & & \\
\hline$<70$ & $70(66.78 \%)$ & $64(71.91 \%)$ & $6(37.50 \%)$ & & \\
\hline
\end{tabular}

Bolded values indicate significant group differences at $p<0.05$

$A D E C$-V Autism Detection in Early Childhood-Virtual, ADI-R Autism Diagnostic Interview-Revised, CARS-2 Childhood Autism Rating Scale2nd Edition

${ }^{a}$ McNemar's Chi-Squared Test

${ }^{b}$ Based on the Cognitive scale of the Developmental Profile-3rd, or 4th edition parent interview

\section{Childhood Autism Rating Scale, Second Edition (CARS-2; Schopler et al., 2010)}

The CARS-2 is a standardized clinician observational tool for autism symptoms in children. It is a clinician report form that is intended for use after completing a behavioral observation and parent interview to guide clinician decision making. The Standard Version (CARS2-ST), appropriate for children six years or younger, with communication delays, or with IQs at or below 79, was used for the current study. Higher scores indicate more pronounced ASD characteristics. The CARS-2-ST has strong internal consistency ( $\alpha=0.93$; Schopler et al., 2010).

\section{Developmental Profile, Third and Fourth Editions (DP-3, Alpern, 2007; DP-4, Alpern, 2020)}

The DP-3 and DP-4 are norm-referenced assessments that provide standardized information about development functioning. The DP-3 was used for participants seen prior to the publication of the fourth edition in 2020, at which time the DP-4 began being used for evaluations. The item response 
format is a binary yes/no answer for different skill areas. Higher scores indicate better developmental skills. For the current study, the Parent/Caregiver Interview Form was administered via interview and the Cognitive scale was used as an estimate of cognitive level.

\section{Procedure}

The study was approved by the hospital internal review board (IRB). Data for this study were based on clinical evaluations for ASD conducted at the child development center of a large hospital in the Midwest United States. Children were initially referred for an ASD evaluation from sources such as state early intervention programs and pediatricians, or they were self-referred due to developmental concerns. Referred families then attended a diagnostic intake appointment, where an unstructured screening interview and brief observation of the child was completed by trained allied healthcare professionals. Children considered at-risk for ASD based on their intake were referred for a comprehensive diagnostic assessment, which typically occurred 6-9 months following this initial triaging appointment. Referrals were made either for an interdisciplinary evaluation with a licensed psychologist, speech-language pathologist, and medical professional, or a targeted single discipline assessment conducted by a licensed clinical psychologist if there were few concerns of comorbid medical concerns and the child had previously received a speech language evaluation. Psychometricians provided assistance in both appointment types. Psychometricians were Bachelor's level clinicians who received a high degree of training in the administration of developmental and ASD-specific assessments (i.e., CARS, ADOS-2) under the supervision of licensed psychologists. These evaluations were conducted via telehealth over a videoconferencing platform (i.e., Zoom ${ }^{\mathrm{TM}}$ ) due to COVID19 pandemic restrictions on in-person meetings. ASD-specific evaluation procedures were consistent across single and interdisciplinary assessments.

ASD evaluation procedures involved administration of the ADEC-V, with the psychometrician guiding the parent through administration of individual ADEC tasks with the child while the psychologist (and speech therapist and medical provider during interdisciplinary appointments) observed. The psychometrician then scored the ADEC-V separately from the diagnostic team to keep them masked of resulting risk scores. All psychometricians completed rigorous training on administration and scoring of the ADECV. First, a virtual training was completed with the ADEC's original author (R.L.Y.) on remote administration and scoring. Next, psychometricians completed inter-rater reliability checks until they achieved $90 \%$ reliability with the study leads (E.M.K. \& C.M.A.) who had previously established reliability in ADEC administration.
The psychologist administered the ADI-R modified toddler algorithm items either at the same visit or at a separate visit occurring within a week of the ADEC-V administration, and the psychologist or psychometrician completed the DP-3 or DP-4 interview, depending on when the appointment occurred in relation to publication of the DP-4. The CARS-2 was then rated based on a combination of semi-structured observation as well as parent report of information.

\section{Analytic Plan}

All analyses and figures were generated using R 4.0.5 (R Core Team, 2021). Analytic plan and hypotheses were preregistered with the Center for Open Science OSF registries (Stephenson et al., 2021).

Hypothesis 1 Diagnostic and Predictive Validity. Diagnosis accuracy analyses consisted of computing sensitivity, specificity, and negative and positive predictive values (NPV, PPV) for ADEC-V Total scores. A Receiver Operating Characteristic curve was also created for ADEC-V Total scores to produce area under the curve (AUC) values. Additionally, multiple logistic regressions predicting final diagnosis were calculated, entering ADEC-V and ADI-R scores as primary predictors while controlling for age (in years) and estimated cognitive ability (delayed vs. intact based on DP-3/DP-4 scores).

Hypothesis 2 Concurrent Validity. Pearson correlation coefficients were computed for ADEC-V Total scores with CARS-2 Total scores and ADI-R Total scores.

Hypothesis 3 Internal Consistency Reliability and Item Analysis. Internal consistency of all ADEC-V items was calculated using McDonald's omega and Cronbach's alpha. We then investigated the relative functioning of each item by calculating item discrimination index scores for each ADEC-V item to determine if alternative cut-off points may function more effectively when the tool is administered in virtual contexts.

\section{Results}

\section{Hypothesis 1—Diagnostic and Predictive Validity}

A summary of the sensitivity, specificity, PPV, and NPV values across various ADEC-V scores are included in Table 2. The recommended screening cutoff of 11 resulted in improved sensitivity (0.93) but significantly decreased specificity (0.44). A score of 14 on the ADEC-V (corresponding to the ADEC cutoff for "high risk of ASD") resulted in a balance of sensitivity (0.82) and specificity (0.78). The AUC 
Table 2 ADEC-V diagnostic accuracy

\begin{tabular}{|c|c|c|c|c|c|c|c|c|}
\hline ADEC-V score & True positives & False positives & True negatives & False negatives & Sensitivity & Specificity & PPV & NPV \\
\hline$>29$ & 0 & 0 & 18 & 103 & 0 & 1 & - & 0.15 \\
\hline 29 & 1 & 0 & 18 & 102 & 0.01 & 1 & 1 & 0.15 \\
\hline 28 & 3 & 0 & 18 & 100 & 0.03 & 1 & 1 & 0.15 \\
\hline 27 & 5 & 0 & 18 & 98 & 0.05 & 1 & 1 & 0.16 \\
\hline 26 & 9 & 0 & 18 & 94 & 0.09 & 1 & 1 & 0.16 \\
\hline 25 & 16 & 0 & 18 & 87 & 0.16 & 1 & 1 & 0.17 \\
\hline 24 & 24 & 0 & 18 & 79 & 0.23 & 1 & 1 & 0.19 \\
\hline 23 & 29 & 0 & 18 & 74 & 0.28 & 1 & 1 & 0.2 \\
\hline 22 & 36 & 0 & 18 & 67 & 0.35 & 1 & 1 & 0.21 \\
\hline 21 & 46 & 0 & 18 & 57 & 0.45 & 1 & 1 & 0.24 \\
\hline 20 & 54 & 0 & 18 & 49 & 0.52 & 1 & 1 & 0.27 \\
\hline 19 & 60 & 0 & 18 & 43 & 0.58 & 1 & 1 & 0.3 \\
\hline 18 & 61 & 0 & 18 & 42 & 0.59 & 1 & 1 & 0.3 \\
\hline 17 & 69 & 2 & 16 & 34 & 0.67 & 0.89 & 0.97 & 0.32 \\
\hline 16 & 75 & 3 & 15 & 28 & 0.73 & 0.83 & 0.96 & 0.35 \\
\hline 15 & 80 & 4 & 14 & 23 & 0.78 & 0.78 & 0.95 & 0.38 \\
\hline 14 & 84 & 4 & 14 & 19 & 0.82 & 0.78 & 0.95 & 0.42 \\
\hline 13 & 89 & 5 & 13 & 14 & 0.86 & 0.72 & 0.95 & 0.48 \\
\hline 12 & 93 & 7 & 11 & 10 & 0.9 & 0.61 & 0.93 & 0.52 \\
\hline 11 & 96 & 10 & 8 & 7 & 0.93 & 0.44 & 0.91 & 0.53 \\
\hline 10 & 98 & 12 & 6 & 5 & 0.95 & 0.33 & 0.89 & 0.55 \\
\hline 9 & 100 & 13 & 5 & 3 & 0.97 & 0.28 & 0.88 & 0.63 \\
\hline 8 & 101 & 14 & 4 & 2 & 0.98 & 0.22 & 0.88 & 0.67 \\
\hline 7 & 101 & 15 & 3 & 2 & 0.98 & 0.17 & 0.87 & 0.6 \\
\hline 6 & 101 & 16 & 2 & 2 & 0.98 & 0.11 & 0.86 & 0.5 \\
\hline 5 & 101 & 17 & 1 & 2 & 0.98 & 0.06 & 0.86 & 0.33 \\
\hline 4 & 102 & 18 & 0 & 1 & 0.99 & 0 & 0.85 & 0 \\
\hline 3 & 103 & 18 & 0 & 0 & 1 & 0 & 0.85 & - \\
\hline
\end{tabular}

$A D E C-V$ Autism Detection in Early Childhood-Virtual, $P P V$ positive predictive value, $N P V$ negative predictive value

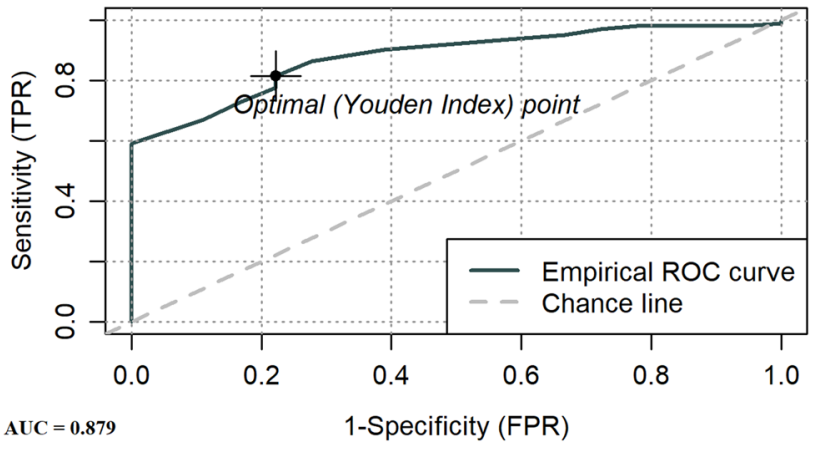

Fig. 1 Receiver operating curve for the ADEC-V

was 0.879 , indicating good overall diagnostic accuracy (see Fig. 1).

Logistic regression results indicated that both ADECV [OR $1.33(1.16-1.60), p<0.001]$ and ADI-R [OR 1.36 $(1.15-1.68), p<0.001]$ scores individually predicted variability in final clinical diagnosis. Age and estimated cognitive level were not significant predictors and did not substantively change the results (see Tables 3,4).

\section{Hypothesis 2-Concurrent Validity}

ADEC-V scores were modestly correlated with ADI-R scores $(r=0.26, p<0.01)$ and strongly correlated with CARS-2 scores $(r=0.70, p<0.001)$. The correlation between ADI-R and CARS- 2 scores was moderate $(r=0.45$, $p<0.001)$. Age was negatively correlated with ADEC-V scores $(r=-0.25, p<0.01)$, but not ADI-R or CARS-2 scores $(r=-0.04$ and -0.08 , respectively).

\section{Hypothesis 3-Internal Consistency Reliability and Item Analysis}

ADEC-V items had acceptable levels of internal consistency (McDonald's $\omega=0.75$, Cronbach's $\alpha=0.77$ ). Item 
Table 3 ADEC-V predicting diagnosis logistic regression

\begin{tabular}{|c|c|c|c|c|c|c|}
\hline \multirow[t]{2}{*}{ Predictors } & \multicolumn{3}{|c|}{ Predicting diagnosis } & \multicolumn{3}{|c|}{ Predicting diagnosis with covariates } \\
\hline & Odds ratios & CI & $p$ & Odds ratios & CI & $p$ \\
\hline (Intercept) & 0.08 & $0.01-0.43$ & 0.006 & 0 & $0.00-0.42$ & 0.026 \\
\hline ADEC-V & 1.33 & $1.19-1.55$ & $<0.001$ & 1.34 & $1.16-1.60$ & $<0.001$ \\
\hline Age (months) & & & & 1.08 & $0.98-1.20$ & 0.153 \\
\hline Cognitive Level & & & & 1.37 & $0.36-5.20$ & 0.643 \\
\hline Observations & 121 & & & 105 & & \\
\hline R2 Tjur & 0.272 & & & 0.263 & & \\
\hline
\end{tabular}

$A D E C-V$ Autism Detection in Early Childhood-Virtual

\begin{tabular}{|c|c|c|c|c|c|c|}
\hline \multirow[t]{2}{*}{ Predictors } & \multicolumn{3}{|c|}{ Predicting diagnosis } & \multicolumn{3}{|c|}{ Predicting diagnosis with covariates } \\
\hline & Odds ratios & CI & $p$ & Odds ratios & CI & $p$ \\
\hline (Intercept) & 0 & $0.00-0.03$ & $<0.001$ & 0 & $0.00-0.01$ & 0.005 \\
\hline ADEC & 1.33 & $1.16-1.60$ & $<0.001$ & 1.45 & $1.19-1.88$ & 0.001 \\
\hline ADI-R & 1.36 & $1.15-1.68$ & 0.001 & 1.37 & $1.14-1.73$ & 0.002 \\
\hline Age (months) & & & & 1.12 & $0.99-1.30$ & 0.105 \\
\hline Cognitive Level & & & & 1.03 & $0.98-1.09$ & 0.335 \\
\hline Observations & 113 & & & & 100 & \\
\hline R2 Tjur & 0.495 & & & & 0.467 & \\
\hline
\end{tabular}

$A D E C-V$ Autism Detection in Early Childhood-Virtual, ADI-R Autism Diagnostic Interview-Revised
Table 4 ADEC-V and ADI-R predicting diagnosis logistic regression
Discrimination Indices for three ADEC-V items were low: Items 3 ("Stereotypical Behavior", 0.05), 9 ("Response to Everyday Sounds", - 0.01), and 14 ("Nestling into Caregiver", 0.06). Removal of these three low-performing items improved internal consistency (McDonald's $\omega=0.82$, Cronbach's $\alpha=0.82$ ), but did not significantly improve diagnostic accuracy $(\mathrm{AUC}=0.877$; see Supplementary Table 1).

\section{Discussion}

Restrictions put in place due to the COVID-19 pandemic necessitated the rapid design and implementation of telehealth assessment models for ASD to prevent already long waitlists from growing exponentially during this health crisis. The telehealth model implemented in the current study was designed to include components deemed essential to a comprehensive evaluation for ASD, including a caregiver interview of ASD symptoms and broader development, and direct observation of behavior indicating the presence of ASD. The virtual version of the ADEC (ADEC-V) was adapted from the original ADEC (Young, 2007), an interactive screener of ASD symptoms. The ADEC-V was used in the current study to structure a direct behavioral observation for children under the age of 4 years old. The ADEC was chosen to be adapted as a virtual assessment tool given the staff's familiarity with the tool in the context of the need to make a quick switch to telehealth assessment, as there were no validated telehealth behavior observation tools for ASD available. The ADEC already had well-established psychometric properties (Young, 2007) and strong research support for use to accelerate and inform clinical screening for ASD (Hedley et al., 2015; Young \& Nah, 2016), with its scores showing very good reliability with the ADOS-2 (Bellando et al., 2018; Hedley et al., 2015), and the author supported the adaption to a virtual format. The measure was a particularly good fit to be adapted to a virtual format for several reasons: clear and concrete instructions, which were found to be readily adapted to parent-led activities that could be completed reliably with clinician coaching; materials needed for administration were likely to be available in most homes with young children; relative low cost, as the only direct cost was the manual and scoring protocols.

In the current study, the ADEC-V was found to have good diagnostic accuracy and predictive validity. The original recommended cutoff score of 11 (designated as "Moderate Risk" on the ADEC) allowed for high sensitivity (0.94) but poorer specificity (0.44). A cutoff of 14 (designated as "High Risk" for the ADEC) resulted in a better balance of sensitivity $(0.82)$ and specificity $(0.78)$. These results are similar to other studies that examined the use of the ADEC in clinical samples (Dix et al., 2015; Hedley et al., 2015), suggesting a higher cutoff may be more appropriate in clinical populations where improved specificity is desired.

Our results indicated that both the ADEC-V and the ADI-R contributed significantly and separately to 
predictive validity of final diagnoses, suggesting that the combination of a structured measure of behavior captured virtually and led by caregivers under the guidance of a clinician, along with the information gathered through a structured caregiver interview, are both important components of a comprehensive evaluation, independent of a child's chronological age or cognitive level. This finding is particularly strengthened by good concurrent validity scores that were demonstrated between the ADEC-V and both the CARS- 2 and the ADI-R. As would be expected, the relationship was much stronger between the CARS-2 and the ADEC-V, as both were completed primarily based on direct observation of the child by telehealth, although the CARS-2 does allow for caregiver report to be considered. The relationship between the ADI-R and the ADEC-V was understandably weaker, as they are capturing information about ASD symptoms from two different sources. Previous research has shown similar poor agreement between the ADI-R and direct behavior observation measures, such as the ADOS-2 and CARS-2 (Wiggins \& Robins, 2008). This may be because direct observation measures, such as the ADEC-V, allow the examiner to see information that a caregiver might miss or interpret differently (e.g., scripted language that a caregiver mistakes as advanced learning of early academic concepts). Interview measures, such as the ADI-R, allow for a broader sampling of behavior that would be difficult to capture in a single structured observation (e.g., overly rigid routines that happen at different times of day; poor interactions with peers), but are balanced by the possible inaccuracies that accompany caregiver reported information. These findings are in line with previous research stating that best clinical practice in ASD evaluation involves caregiver interviewing combined with observation of child behavior (Huerta $\&$ Lord 2012). Therefore, it is strongly recommended that the ADEC-V be used in combination with a standardized interview measure such as the ADI-R.

Scores on the ADEC-V were slightly negatively correlated with age, suggesting that older children tended to get lower scores on the ADEC-V. While age was not a significant predictor of final diagnosis, it should be kept in mind that the ADEC was originally designed for children up to 3 years old, but the current study expanded the age range to 4 years old. This was due to current clinical utility via telehealth for children up through preschool-age, particularly those with speech and language delays and in the absence of other available behavioral measures that could be administered over the video platform. The slight negative correlation is likely due to ADEC-V items targeting behaviors that are most indicative of ASD prior to the age of three, such as deficits in pointing, showing, and eye contact, and may not as easily identify older children with milder symptom presentations (Barbaro \& Dissanayake, 2013). Based on these results it is suggested that caution be used when using this measure with children 3-4 years old.

Overall, the ADEC-V showed good internal consistency, although there were three items that did not discriminate well between children with and without ASD (i.e., "Stereotypical Behavior", "Response to Everyday Sounds", "Nestling into Caregiver"). These items were also found to be among the poorest functioning in terms of discriminative validity in other investigations of the ADEC (Nah et al., 2019; Nevill et al., 2019). These behaviors could be explored in further studies to see if similar patterns are found. It should be noted that while removing these items improved internal consistency, it did not improve overall diagnostic accuracy.

\section{Strengths, Limitations, and Future Directions}

A primary strength of this preliminary feasibility and validation study was a reasonable sample size that included a high representation of children from Black and other racial minority backgrounds, and an overall racial breakdown that is comparable to national estimates (U.S. Census Bureau, 2021). The study team also completed a rigorous training procedure on the ADEC-V with the tools original author to ensure validity of scoring procedures. Given that the ADEC$\mathrm{V}$ is a brief assessment tool with an easy scoring system, all administrators were able to achieve reliability within three practice sessions. Further, the ADEC-V does not require a standard set of items to use, so it was easily implemented within the family home by using commonly available toys and items already in the family's possession. Overall, this study demonstrated good external validity by assessing the utility and psychometric properties of the ADEC-V in an applied clinical setting by implementing it as part of standard clinical care.

One potential limitation of this sample was that all children had been referred for assessment due to developmental concerns and had completed a diagnostic interview, which screened out children for whom ASD was not a primary concern. Therefore, ASD was the primary concern for all children included in the current study, indicating a potential sample bias. The ADEC-V performed reasonably well in this sample, but this may have explained why specificity was low when applying the recommended cutoff score. Combining typically developing children with those with developmental delays and ASD concerns has been shown to improve specificity in similar measures (Kim \& Lord, 2012b; Nah et al., 2014), and is an important direction for future work with the ADEC-V. Future research could also examine the use of the ADEC-V in a screening context to assess whether it is more effective than the clinical interview alone. Nonetheless, it is important to note that the ADEC-V was specifically used in the current setting to provide a structured 
behavioral assessment of children in a remote setting in order to improve the clinicians' ability to make a diagnostic decision. This is consistent with current ASD diagnostic recommendations to incorporate both behavioral observation and clinical interview to form a diagnostic impression (Huerta \& Lord, 2012; Zwaigenbaum et al., 2009).

The present study was also limited by not collecting data from caregivers and clinicians concerning feasibility of the ADEC-V. This is important given that the evaluation modality is novel for most clinicians and it places more pressure on parents due to them being required to administer the instrument under remote instruction. Future research should gather information on parent and clinician perspective regarding their role in administering the ADEC-V.

Due to COVID restrictions, another limitation of the current study was that it was not possible to complete direct developmental/early cognitive testing, as standardized telehealth measures to assess these skills directly do not yet exist. Finally, as all study procedures were completed as part of standard clinical care, it was not within the scope of this study to bring back participants for independent diagnostic verification. Once COVID-19 restrictions can be fully lifted, further analysis of the ADEC-V can be completed, comparing its performance to the standard in-clinic assessment, including an ADOS-2 or ADEC and direct developmental assessment, completed by an independent clinician.

It is also important to note that there are cases where assessment by telehealth is not appropriate, and where lengthier, more in-depth, in-clinic evaluations are necessary to make an accurate diagnosis and appropriate treatment recommendations. Future research should, therefore, also focus on identifying characteristics that could help triage families to the type of evaluation that would best fit their needs.

\section{Conclusion}

This study provides initial preliminary support for the use of the ADEC-V in diagnostic assessment of ASD over telehealth. It was found to accurately identify ASD symptoms in young children, and paired well with the ADI-R as major components of a comprehensive telehealth ASD assessment model. Utilizing comparable models of care that can be completed via telehealth increases expansion of health care by reducing barriers such as geographical location away from major healthcare centers, poor access to transportation, and the inability to miss work or find additional childcare to be able to attend medical appointments. The ADEC-V also has the potential to increase access to ASD evaluations by reducing long waitlists, as it allows for shorter administration times, reduced visit times in general (e.g., less time needed for checking in and out), decreased need for exam room space, etc. Telehealth models of care will also improve the agility of our healthcare systems during times of crisis, such as the current case of the global pandemic. The ADEC-V is a promising measure that could be used to further expand telehealth assessment possibilities for young children suspected of ASD, leading to expanded access to services among a particularly vulnerable population. The current preliminary validation study suggests that the measure is appropriate for use in a busy clinical setting with a diverse sample. Further investigation into the performance of the ADEC-V in non-clinically referred populations, as well as larger studies comparing how the diagnostic accuracy of telehealth models of ASD assessment using the ADEC-V to traditional in-clinic ASD assessment models would be important next steps in this line of research.

Supplementary Information The online version contains supplementary material available at https://doi.org/10.1007/s10803-022-05433-1.

Acknowledgments We would like to thank the interdisciplinary teams at the Child Development Center who participated in the transition to telehealth for their flexibility and willingness to try something new. We would also like to thank the families who have provided invaluable feedback on the model.

Author Contributions All authors contributed to the study conception and design. Material preparation and data collection were performed by EMK, CMA. Data analysis was performed by KGS. The first draft of the manuscript was written by EMK, CMA, KGS, and COB. All authors commented on previous versions of the manuscript. All authors read and approved the final manuscript.

Funding The authors received no financial support for the research, authorship, and/or publication of this article. Darren Hedley is supported by a Suicide Prevention Australia National Suicide Prevention Research fellowship.

\section{Declarations}

Conflict of interest Robyn L. Young declares that Flinders University receives funding from ACER press for sales of the ADEC. The remaining authors declare no other actual or potential conflict of interest.

Ethical Approval This study was performed in line with the principles of the Declaration of Helsinki. Approval was granted by the Ethics Committee of Nationwide Children's Hospital (STUDY00001524 \& STUDY00001171).

Consent to Participate The study was deemed as no greater than minimal risk by the Ethics Committee of Nationwide Children's Hospital, so a waiver of consent documentation was granted for all participants who completed assessments before the study began (STUDY00001171) and a Waiver of HIPAA authorization was completed with all participants recruited after the study began (STUDY00001524).

\section{References}

Alpern, G. (2007). Developmental profile, third edition (DP-3). Western Psychological Services. https://www.researchconnections.org/ childcare/resources/26853. Accessed 3 July 2021.

Alpern, G. (2020). Developmental profile, fourth edition (DP-4). Western Psychological Services. 
Barbaro, J., \& Dissanayake, C. (2013). Early markers of autism spectrum disorders in infants and toddlers prospectively identified in the social attention and communication study. Autism, 17(1), 64-86. https://doi.org/10.1177/1362361312442597

Bellando, J., Schulz, E., Fussell, J.J., Chang, D., Pearson, K., Jernigan, S., Oswalt, J., Oswalt, K., Reid, T., Whitaker, B., Carey, R., Lopez, M., \& Goudie, A. (2018). Community Based Autism Liaison \& Treatment Project (CoBALT): Decreasing wait time and increasing access to services. Poster Presentation at the International Society for Autism Research 2018 Annual Meeting, Rotterdam, Netherlands

Berger, N. I., Wainer, A. L., Kuhn, J., Bearss, K., Attar, S., Carter, A. S., Ibanez, L. V., Ingersoll, B. R., Neiderman, H., Scott, S., \& Stone, W. L. (2021). Characterizing available tools for synchronous virtual assessment of toddlers with suspected autism spectrum disorder: A brief report. Journal of Autism and Developmental Disorders. https://doi.org/10.1007/s10803-021-04911-2

Corona, L., Hine, J., Nicholson, A., Stone, C., Swanson, A., Wagner, L., Weitlauf, A. S., \& Warren, Z. (2020a). TELE-ASD-PEDS: A telemedicine-based ASD evaluation tool for toddlers and young children. Vanderbilt University Medical Center. https:// vkc.vumc.org/vkc/triad/tele-asd-peds. Accessed 23 Aug 2020.

Corona, L. L., Weitlauf, A. S., Hine, J., Berman, A., Miceli, A., Nicholson, A., Stone, C., Broderick, N., Francis, S., Juárez, A. P., Vehorn, A., Wagner, L., \& Warren, Z. (2020b). Parent perceptions of caregiver-mediated telemedicine tools for assessing autism risk in toddlers. Journal of Autism and Developmental Disorders. https://doi.org/10.1007/s10803-020-04554-9

Dix, L., Fallows, R., \& Murphy, G. (2015). Effectiveness of the ADEC as a Level 2 screening test for young children with suspected autism spectrum disorders in a clinical setting. Journal of Intellectual \& Developmental Disability, 40(2), 179-188. https://doi. org/10.3109/13668250.2015.1014323

Gordon-Lipkin, E., Foster, J., \& Peacock, G. (2016). Whittling down the wait time: Exploring models to minimize the delay from initial concern to diagnosis and treatment of autism spectrum disorder. Pediatric Clinics of North America, 63(5), 851-859. https://doi.org/10.1016/j.pcl.2016.06.007

Hedley, D., Nevill, R. E., Monroy-Moreno, Y., Fields, N., Wilkins, J., Butter, E., \& Mulick, J. A. (2015). Efficacy of the ADEC in identifying autism spectrum disorder in clinically referred toddlers in the US. Journal of Autism and Developmental Disorders, 45(8), 2337-2348. https://doi.org/10.1007/ s10803-015-2398-5

Hedley, D., Young, R., Angelica, M., Gallegos, J., \& Marcin Salazar, C. (2010). Cross-cultural evaluation of the autism detection in early childhood (ADEC) in Mexico. Autism, 14(2), 93-112. https://doi. org/10.1177/1362361309347676

Huerta, M., \& Lord, C. (2012). Diagnostic evaluation of autism spectrum disorders. Pediatric Clinics of North America, 59(1), 103-xi. https://doi.org/10.1016/j.pcl.2011.10.018

Jang, J., White, S. P., Esler, A. N., Kim, S. H., Klaiman, C., Megerian, J. T., Morse, A., Nadler, C., \& Kanne, S. M. (2021). Diagnostic evaluations of autism spectrum disorder during the COVID-19 pandemic. Journal of Autism and Developmental Disorders. https://doi.org/10.1007/s10803-021-04960-7

Kawashima, H., Dissanayake, C., Hedley, D., Kezuka, E., \& Okuno, M. (2021). Validation of autism detection in early childhood-Japanese $(A D E C-J)$. Poster presented at the International Society for Autism Research Annual Meeting, Virtual, May 3-7

Kim, S. H., \& Lord, C. (2012a). New autism diagnostic interviewrevised algorithms for toddlers and young preschoolers from 12 to 47 months of age. Journal of Autism and Developmental Disorders, 42(1), 82-93. https://doi.org/10.1007/s10803-011-1213-1

Kim, S. H., \& Lord, C. (2012b). Combining information from multiple sources for the diagnosis of autism spectrum disorders for toddlers and young preschoolers from 12 to 47 months of age. Journal of Child Psychology and Psychiatry, and Allied Disciplines, 53(2), 143-151. https://doi.org/10.1111/j.1469-7610.2011.02458.x

Kryszak, E. M., \& Albright, C. (2020). How do we adapt in a time of crisis? APA Division 33 Newsletter, 46(1), 4.

Le Couteur, A., Lord, C., \& Rutter, M. (2003). ADI-R: Autism diagnostic interview-revised. Western Psychological Services.

Lecavalier, L., Aman, M. G., Scahill, L., McDougle, C. J., McCracken, J. T., Vitiello, B., Tierney, E., Arnold, L. E., Ghuman, J. K., Loftin, R. L., Cronin, P., Koenig, K., Posey, D. J., Martin, A., Hollway, J., Lee, L. S., \& Kau, A. S. M. (2006). Validity of the autism diagnostic interview-revised. American Journal on Mental Retardation, 111(3), 199-215. https://doi.org/10.1352/08958017(2006)111[199:VOTADI]2.0.CO;2

Lord, C., Holbrook, A., Dow, D., Byrne, K., Grzadzinski, R., \& Sterett, K. (2020). Brief observation of symptoms of autism (BOSA) [unpublished manual]. Western Psychological Services.

Lord, C., Rutter, M., DiLavore, P., Risi, S., Gotham, K., \& Bishop, S. (2012). Autism diagnostic observation schedule-2nd edition (ADOS-2). Western Psychological Corporation.

Miller, J. (2020). ASD-DIAL: Diagnostic interview and activitiesLifespan, version 2. Children's Hospital of Philadelphia

Nah, Y.-H., Young, R. L., \& Brewer, N. (2019). Development of a brief version of the autism detection in early childhood. Autism, 23(2), 494-502. https://doi.org/10.1177/1362361318757563

Nah, Y.-H., Young, R. L., Brewer, N., \& Berlingeri, G. (2014). Autism detection in early childhood (ADEC): Reliability and validity data for a level 2 screening tool for autistic disorder. Psychological Assessment, 26(1), 215-226. https://doi.org/10.1037/a0034472

Nevill, R. E., Hedley, D., \& Uljarević, M. (2019). Brief report: Replication and validation of the brief autism detection in early childhood (BADEC) in a clinical sample. Journal of Autism and Developmental Disorders, 49(11), 4674-4680. https://doi.org/10.1007/ s10803-019-04153-3

Nickel, R. (2020). The observation of play screener: Home edition. Oregon Health \& Science University.

R Core Team. (2021). R: A language and environment for statistical computing (4.0.5) [Computer software]. $\mathrm{R}$ foundation for statistical computing. https://www.R-project.org/. Accessed 18 July 2021.

Schopler, E., Van Bourgondien, M. E., Wellman, G. J., \& Love, S. R. (2010). CARS-2: Childhood autism rating scale. Western Psychological Services.

Sidjaja, F. F. (2015). Assessment and diagnosis of autism in developing countries: The Indonesian adaptation of Autism Detection in Early Childhood (ADEC) [Doctoral thesis, The University of Queensland]

Stephenson, K., Burns, C., Hedley, D., Young, R. L., Nevill, R., Mulick, J. A., Albright, C., Kryszak, E., \& Butter, E. (2021, May 14). AVABO-ADEC Autism Telehealth. osf.io/83dfq.

Stone, W. L., Coonrod, E. E., \& Ousley, O. Y. (2000). Brief report: Screening tool for autism in two-year-olds (STAT): Development and preliminary data. Journal of Autism and Developmental Disorders, 30(6), 607-612. https://doi.org/10.1023/a:1005647629002

U.S. Census Bureau. (2021). U.S. Census Bureau QuickFacts: United States. https://www.census.gov/quickfacts/fact/table/US/PST04 5219. Accessed 15 Dec 2020.

Wagner, L., Corona, L. L., Weitlauf, A. S., Marsh, K. L., Berman, A. F., Broderick, N. A., Francis, S., Hine, J., Nicholson, A., Stone, C., \& Warren, Z. (2020). Use of the TELE-ASD-PEDS for autism evaluations in response to COVID-19: Preliminary outcomes and clinician acceptability. Journal of Autism and Developmental Disorders. https://doi.org/10.1007/s10803-020-04767-y

Wagner, L., Weitlauf, A. S., Hine, J., Corona, L. L., Berman, A. F., Nicholson, A., Allen, W., Black, M., \& Warren, Z. (2021). Transitioning to telemedicine during COVID-19: Impact on perceptions 
and use of telemedicine procedures for the diagnosis of autism in toddlers. Journal of Autism and Developmental Disorders. https:// doi.org/10.1007/s10803-021-05112-7

Wetherby, A., Woods, J., Allen, L., Cleary, J., Dickinson, H., \& Lord, C. (2004). Early indicators of autism spectrum disorders in the second year of life. Journal of Autism and Developmental Disorders, 34, 473-493.

Wiggins, L. D., \& Robins, D. L. (2008). Brief report: Excluding the ADI-R behavioral domain improves diagnostic agreement in toddlers. Journal of Autism and Developmental Disorders, 38(5), 972-976. https://doi.org/10.1007/s10803-007-0456-3

Young, R. L. (2007). Autism Detection in Early Childhood (ADEC) Manual. ACER Press. https://dspace.flinders.edu.au/xmlui/handle/2328/13414. Accessed 1 July 2020.

Young, R. L., \& Nah, Y.-H. (2016). Examining Autism detection in early childhood (ADEC) in the early identification of young children with autism spectrum disorders (ASD). Australian Psychologist, 51(4), 261-271. https://doi.org/10.1111/ap.12223

Zander, E., Willfors, C., Berggren, S., Coco, C., Holm, A., Jifält, I., Kosieradzki, R., Linder, J., Nordin, V., Olafsdottir, K., \& Bölte, S. (2017). The interrater reliability of the autism diagnostic interview-revised (ADI-R) in clinical settings. Psychopathology, 50(3), 219-227. https://doi.org/10.1159/000474949

Zwaigenbaum, L., Bryson, S., Lord, C., Rogers, S., Carter, A., Carver, L., Chawarska, K., Constantino, J., Dawson, G., Dobkins, K., Fein, D., Iverson, J., Klin, A., Landa, R., Messinger, D., Ozonoff, S., Sigman, M., Stone, W., Tager-Flusberg, H., \& Yirmiya, N. (2009). Clinical assessment and management of toddlers with suspected autism spectrum disorder: Insights from studies of high-risk infants. Pediatrics, 123(5), 1383-1391. https://doi.org/ 10.1542/peds.2008-1606

Zwaigenbaum, L., \& Warren, Z. (2021). Commentary: Embracing innovation is necessary to improve assessment and care for individuals with ASD: A reflection on Kanne and Bishop (2020). Journal of Child Psychology and Psychiatry, 62(2), 143-145. https://doi.org/10.1111/jcpp.13271

Publisher's Note Springer Nature remains neutral with regard to jurisdictional claims in published maps and institutional affiliations. 